Research paper

\title{
Suicide mortality and use of psychotropic drugs in patients hospitalized due to bipolar disorder: A Finnish nationwide cohort study
}

\author{
Diego Antolín-Concha ${ }^{\mathrm{a}, *, *}$, Markku Lähteenvuo ${ }^{\mathrm{a}, *, *}$, Pia Vattulainen ${ }^{\mathrm{e}}$, Antti Tanskanen ${ }^{\mathrm{a}, \mathrm{b}, \mathrm{c}}$, \\ Heidi Taipale $^{\mathrm{a}, \mathrm{b}, \mathrm{d}}$, Eduard Vieta ${ }^{\mathrm{f}}$, Jari Tiihonen ${ }^{\mathrm{a}, \mathrm{b}, \mathrm{g}}$ \\ ${ }^{a}$ University of Eastern Finland, Department of Forensic Psychiatry, Niuvanniemi Hospital, Finland (Antolín-Concha, Lähteenvuo, Tanskanen, Taipale, Tïhonen) \\ ${ }^{\mathrm{b}}$ Department of Clinical Neuroscience, Karolinska Institutet, Stockholm, Sweden (Tanskanen, Taipale, Tuihonen) \\ ${ }^{\mathrm{c}}$ National Institute for Health and Welfare, Impact Assessment Unit, Helsinki, Finland (Tanskanen) \\ ${ }^{\mathrm{d}}$ School of Pharmacy, University of Eastern Finland, Kuopio, Finland (Taipale) \\ ${ }^{\mathrm{e}}$ EPID Research Oy, Espoo, Finland (Vattulainen) \\ ${ }_{\mathrm{f}}^{\mathrm{f}}$ Institute of Neurosience, Hospital Clinic, University of Barcelona, IDIBAPS, CIBERSAM, Barcelona, Catalonia, Spain (Vieta) \\ ${ }^{\mathrm{g}}$ Center for Psychiatry Research, Stockholm City Council, Stockholm, Sweden (Tïhonen)
}

\section{A R T I C L E I N F O}

\section{Keywords:}

Pharmacological treatment

Bipolar disorder

Mortality

Suicide

\begin{abstract}
A B S T R A C T
Background: Knowledge on the comparative effectiveness of pharmacological treatments to prevent suicide mortality in bipolar disorder is still lacking.

Methods: We studied the risk of suicide mortality during 1996-2012 among all patients who had been hospitalized due to bipolar disorder in Finland ( $\mathrm{n}=18,018$; mean follow-up time 7.2 years) using nation-wide databases. We used a Cox proportional hazards model. Analyses were adjusted for the effects of time since diagnosis, order of treatments, current use of other treatments, polypharmacy, number of suicidal hospitalizations within 2 year (indicator of inherent risk of relapse), age at index date, sex, and calendar year of index date. In secondary analysis, the first 30 days were omitted from analysis after initiation of a psychopharmacological treatment to control for protopathic bias.

Results: In comparison between use and no use among specific agents, only lithium (HR 0.33, 95\%CI 0.24-0.47, $\mathrm{p}<0.0001$ ) and valproic acid (HR 0.61, 95\%CI 0.48-0.79, $\mathrm{p}=0.0002$ ) were associated with a significantly decreased risk of suicide in bipolar disorder. Lithium showed a $42 \%$ lower risk for suicide mortality compared to valproic acid (HR 0.58, 95\% CI 0.39-0.86, p = 0.007). Hypnotics were associated with a significantly (HR 1.52, 95\%CI 1.22-1.90, $\mathrm{p}=0.0002$ ) higher risk of suicide.

Limitations: Only hospitalized patients were included.

Conclusions: Lithium should be considered as treatment of choice for patients with bipolar disorder who are at high risk for suicide. Hypnotic use among suicidal patients indicates need for close monitoring of these patients.
\end{abstract}

\section{Introduction}

Bipolar disorder is a serious mental disorder that affects around 2-5\% of the general population (Clemente et al., 2015, Angst, 1998, Akiskal et al., 2000). On average, patients with bipolar disorder die approximately 9 years earlier than the general population and 9 - 15\% of them die by suicide (Crump et al., 2013, Medici et al., 2015,
Hayes et al., 2015).

Lithium salts have been the cornerstone of treatment for bipolar disorder during the last decades (Collins and McFarland, 2008). However, in recent years, the usage of other psychotropics has substantially increased (Vieta et al., 2013, Kessing et al., 2016, Karanti et al., 2016, Rhee et al., 2020) on the expense of lithium. In particular, anticonvulsant drugs like valproic acid and antipsychotics have partially

\footnotetext{
Abbreviations: ATC, anatomical therapeutic chemical-classification; CED, cohort entry date; FDR, Benjamini-Hochberg false discovery rate; HR, hazard ratio; CI, confidence interval; PRE2DUP, prescription to drug use periods

Previous presentation: This data has previously been presented at the ECNP2018 (Barcelona, Oct 6 - 10) and ACNP2018 (Hollywood, Florida, USA, Dec 9 - 13) meetings.

* Corresponding authors.

E-mail address: markku.lahteenvuo@niuva.fi (M. Lähteenvuo).

$*=$ equal contribution
} 
replaced lithium as the first-line treatment for this disorder. Other pharmacological options are also often prescribed as a long-term adjuvant therapy or to control some of the symptoms sporadically present in these patients (Vieta et al., 2018). For instance, in a recent Swedish nationwide registry study, a trend towards the decreased use of lithium and increased use of antidepressants was observed in the treatment of bipolar disorder (Carlborg et al., 2015).

As suicide constitutes the second most frequent cause of death after cardiovascular disease and is one of the main causes for the premature mortality observed in patients with bipolar disorder (Ösby et al., 2001), it should be one of the key aspects considered when choosing treatment regimens. Many studies have shown lithium to be an effective treatment for suicidality in bipolar disorder (Kessing et al., 2005, Smith and Cipriani, 2017, Cipriani et al., 2005, Cipriani et al., 2013, Tondo and Baldessarini, 2000, Tondo et al., 2001). However, lithium use harbors a risk for adverse side-effects and discontinuation rates are high, with adverse effects being the most prominent reason for discontinuation (Öhlund et al., 2018). Other mood stabilizers have been shown to be effective as well, but the comparative effectiveness of these treatments remains conflicting (Søndergård et al., 2008, Smith et al., 2009, Song et al., 2017, Ferrer et al., 2014, Collins and McFarland, 2008, Geddes et al., 2010, Oquendo et al., 2011). Also, a large body of the evidence relies on randomized controlled trials and their meta-analyses, from which suicidal patients are often excluded (Baldessarini et al., 2019). Further, studies looking at the comparative effectiveness of different pharmacological groups, such as mood stabilizers, antipsychotics and antidepressants, are scarce (Tondo and Baldessarini, 2018, Marangell et al., 2008), and a recent publication by the International Society for Bipolar Disorders Task Force on Suicide in Bipolar Disorder called for more research on this topic (Schaffer et al., 2015). Due to lack of data on comparative effectiveness, it is possible that medication decisions drift towards choosing medication approaches based on lesser side-effects rather than greater efficacy or antisuicidal properties. It has also been postulated that some medications, such as antidepressants, might increase hypomanic and manic symptoms (Viktorin et al., 2014, Pacchiarotti et al., 2013), which may lead to increased risk of suicide, although data on this topic are still very conflicting. Moreover, in view of the fact that the number of prescriptions for antidepressants has risen, and a portion of patients with bipolar disorder are treated with antidepressants as a monotherapy (Viktorin et al., 2014), which is considered poor practice at least in bipolar I disorder (Vieta, 2014), especially the effect of different antidepressants on suicide mortality also warrants further study.

It is imperative that robust and current information is available on both the efficacy and tolerability of medications. The aim of the present study is to analyze how the use of psychotropic medications is associated with deaths due to suicide in patients hospitalized at least once due to bipolar disorder in Finland. We set out to calculate risk associations for all of the most widely used medication groups, including mood stabilizers, antipsychotics, antidepressants, anxiolytics and hypnotics. To our knowledge, this is one of the largest and most comprehensive observational study on medication risk for bipolar suicide mortality to date.

\section{Methods}

\subsection{Study design and data acquisition}

Finnish nationwide registries were used to combine prospectively collected data in order to conduct a population-based cohort study of patients hospitalized due to (any type of) bipolar disorder. The registers were used to identify the study cohort (patients hospitalized due to bipolar disorder between the years 1987 and 2012), to determine the incidences, durations, and reasons for re-hospitalizations, to obtain information on reimbursed medications dispensed from pharmacies (all psychotropic medications except small packages of anxiolytics were reimbursed in this indication), and to retrieve information on deaths. In Finland, a forensic autopsy is performed whenever someone is found dead, and 1) the death is not known to have been caused by an existing disease or the deceased has not received medical care during his latest course of disease, 2) there is reason to suspect the death might have been caused by an accident, suicide, crime, poisoning, vocational disease, or medical treatment, and 3) the death has been otherwise sudden or unexpected. Therefore, there is reason to assume that most of the deaths by suicide are rightly classified as such.

The Prescription, Hospital Discharge and Causes of death registers and their usage have been described in more detail in our previous pharmacoepidemiological and methodological studies (Tanskanen et al., 2017, Tanskanen et al., 2015, Taipale et al., 2016, Tiihonen et al., 2017). Hospital treatments, deaths, and prescription fillings are documented in these nationwide registries. In Finland, every individual has a unique identification code, which makes it possible to track them even if they would change their name or location of residence.

The current study included every subject ( $\mathrm{n}=18,018$ ) hospitalized at least once with a bipolar disorder diagnosis (ICD-10 diagnoses F3031 were used from 1996 onward and Finnish ICD-9 diagnoses 29622964 and 2967A between 1987-1995, the Finnish ICD-9 coding system somewhat differs from the international one, please see https://www. julkari.fi/bitstream/handle/10024/131850/Tautiluokitus_ICD9_1987. pdf for further details) between January 1:st, 1987 and December 31:st, 2012, who had not been diagnosed with broadly defined schizophrenia during this time period (ICD 10: F20-29, ICD 9: 295, 2971A, 2973A, 2988A, 2989X, 3012C) and who were still alive at the start of the observation period. A total of 25,860 subjects were initially identified, but 7,758 were excluded due to having a schizophrenia diagnosis as described above, 77 excluded due to death before start of observation/ follow-up period, and 7 excluded due to not having any follow-up time (cohort entry at the end of the observation period). Follow-up data for hospitalization periods lasting over 30.5 days was censored.

In order to account for survival bias (selective mortality due to treatment history), we also formed and analyzed a sub-cohort of the study subjects who 1) had never been hospitalized with any mental disorder before cohort entry and 2) had not used psychotropic medication one year prior to cohort entry, namely incident cohort ( $n=2,074)$. These study subjects could be regarded as first-episode patients.

Cohort entry date was set as January 1:st, 1996 for subjects hospitalized due to bipolar disorder between January $1^{\text {st }} 1987$ to December $31^{\text {st }} 1995$, and as the first hospital discharge date for subjects hospitalized for the first time on January $1^{\text {st }} 1996$ or later. The cohorts are described in detail in Table 1.

\subsection{Exposure}

The PRE2DUP method was used to define exposure (use) and nonexposure (non-use) periods for medications (Tanskanen et al., 2017, Tanskanen et al., 2015, Taipale et al., 2016, Tiihonen et al., 2017). PRE2DUP calculates current dose with a sliding average, uses package information such as number of tablets and administration intervals for injections, and takes into account stockpiling when constructing time periods of continuous use. Our previous publications on the validation of the method indicate that PRE2DUP is the most precise method currently available to estimate drug use, and it gives highly accurate drug use periods for most drug classes, especially those meant for long-term use (Tanskanen et al., 2017, Tanskanen et al., 2015, Taipale et al., 2016), As variation in dose is allowed within the method, no artificial grace periods are used. Thus, PRE2DUP models actual medication use on a day-to-day basis. Times when individuals are using a medication (eg. Lithium) or medication group (eg. Mood stabilizers) are compared against times when individuals are not using that same medication (Lithium) or medication group (Mood stabilizers), depending on 
Table 1

Characteristics of study cohorts.

\begin{tabular}{|c|c|c|}
\hline Covariates & \multicolumn{2}{|c|}{ Patient counts (n, \%) } \\
\hline \multicolumn{3}{|l|}{ Gender } \\
\hline female & $9558(53.05 \%)$ & $963(46.43 \%)$ \\
\hline male & $8460(46.95 \%)$ & $1111(53.57 \%)$ \\
\hline \multicolumn{3}{|c|}{ Age in years at cohort entry date } \\
\hline$<30$ & $3345(18.56 \%)$ & $588(28.35 \%)$ \\
\hline $30-49$ & $7121(39.52 \%)$ & $689(33.22 \%)$ \\
\hline $50-69$ & $5577(30.95 \%)$ & $534(25.75 \%)$ \\
\hline$\geq 70$ & $1975(10.96 \%)$ & $263(12.68 \%)$ \\
\hline \multicolumn{3}{|c|}{ Calender year of cohort entry date (n, \%) } \\
\hline 1996-1999 & $5107(28.34 \%)$ & $343(16.54 \%)$ \\
\hline $2000-2003$ & $3102(17.22 \%)$ & $501(24.16 \%)$ \\
\hline $2004-2007$ & $4280(23.75 \%)$ & $573(27.63 \%)$ \\
\hline 2008-2012 & $5529(30.69 \%)$ & $657(31.68 \%)$ \\
\hline \multicolumn{3}{|c|}{ Patients remaining in cohort after censoring hospitalizations longer than 30.5 days } \\
\hline no & $105(0.58 \%)$ & $13(0.63 \%)$ \\
\hline yes & $17913(99.42 \%)$ & $2061(99.37 \%)$ \\
\hline \multicolumn{3}{|c|}{ Time in years since diagnosis at CED } \\
\hline $0-5$ & $16531(91.75 \%)$ & $2074(100 \%)$ \\
\hline $5-10$ & $1472(8.17 \%)$ & $0(0.00 \%)$ \\
\hline$>10$ & $15(0.08 \%)$ & $0(0.00 \%)$ \\
\hline \multicolumn{3}{|c|}{ Time in years since diagnosis at end of follow-up } \\
\hline $0-5$ & $7120(39.52 \%)$ & $870(41.95 \%)$ \\
\hline $5-10$ & $5357(29.73 \%)$ & $669(32.26 \%)$ \\
\hline$>10$ & $5541(30.75 \%)$ & $535(25.80 \%)$ \\
\hline \multicolumn{3}{|c|}{ Use of anxiolytics during follow-up } \\
\hline no & $8580(47.62 \%)$ & $1466(70.68 \%)$ \\
\hline yes & $9438(52.38 \%)$ & $608(29.32 \%)$ \\
\hline \multicolumn{3}{|c|}{ Use of hypnotics during follow-up } \\
\hline no & $8069(44.78 \%)$ & $1306(62.97 \%)$ \\
\hline yes & $9949(55.22 \%)$ & $768(37.03 \%)$ \\
\hline \multicolumn{3}{|c|}{ Use of mood stabilizers during follow-up } \\
\hline no & $4749(26.36 \%)$ & $827(39.87 \%)$ \\
\hline yes & $13269(73.64 \%)$ & $1247(60.13 \%)$ \\
\hline \multicolumn{3}{|c|}{ Use of antidepressants during follow-up } \\
\hline no & $4832(26.82 \%)$ & $1182(56.99 \&)$ \\
\hline yes & $13186(73.18 \%)$ & $892(43.01 \%)$ \\
\hline \multicolumn{3}{|c|}{ Use of antipsychotics follow-up } \\
\hline no & $3413(18.94 \%)$ & $603(29.07 \%$ \\
\hline yes & $14605(81.06 \%)$ & $1471(70.93 \%)$ \\
\hline Total & $18018(100 \%)$ & $2074(100 \%)$ \\
\hline
\end{tabular}

Legend: Characteristics of the study cohorts. Numbers (percentages in parentheses) displayed for both the Total and Incident cohort. CED = cohort entry date.

whether analysis is done on medication or medication group level. Polypharmacy is defined as concurrent use of two or more medications from the same medication group. As not recorded in the register-based data as such, periods of cross-titration and actual polypharmacy (with two or more medications used concomitantly) include somewhat more uncertainty.

Antipsychotics were defined as ATC code N05A, except for N05AN01 (lithium), antidepressants as N06A, mood stabilizers as N03AF, N03AG, N03AX and N05AN01 (lithium), anxiolytics as N05BA, and finally hypnotics as N05C.

\subsection{Outcome}

Suicidal deaths were defined with ICD-10 diagnostic codes X60-X84, as registered in the data from the Causes of death register. Any suicide occurring within 30 days of a start of a hospitalization were censored.

\subsection{Statistical analysis}

An analysis of medication use versus suicide mortality was conducted on both cohorts (main cohort and incident cohort) using the Cox proportional hazards analysis to calculate hazard ratios (HRs) with $95 \%$ confidence intervals (CIs) for use versus non-use of each psychotropic treatment group. The same analysis was done for individual therapeutic agents inside the mood stabilizer, and antidepressant groups. A head-to- head comparison was also completed comparing all mood stabilizers individually against the most widely used mood stabilizer valproate. Omission of the first 30 days from analysis after any initiation of a new psychopharmacological treatment was used to control for protopathic bias on an additional group level analysis. Analyses were adjusted for the effects of time since diagnosis, order of treatments, current use of other treatments, polypharmacy, number of suicidal hospitalizations within a 2-year time period (indicator of inherent risk of relapse), age at index date, sex, and calendar year of index date. The p-values were corrected for multiple comparisons using the Benjamini-Hochberg false discovery rate (FDR) method. Corrected p-values $<0.05$ were considered statistically significant. Nominal p-values are displayed throughout the manuscript, unless otherwise stated.

\subsection{Ethical considerations}

The research project was approved by the Ethics Committee of the Finnish National Institute for Health and Welfare (dated December 4, 2013, 8/2013). Further permissions were granted by pertinent institutional authorities at the Finnish National Institute for Health and Welfare (permission THL/1466/6.02.00/2013), The Social Insurance Institution of Finland (34/522/2013), and Statistics Finland (TK53305-13). 


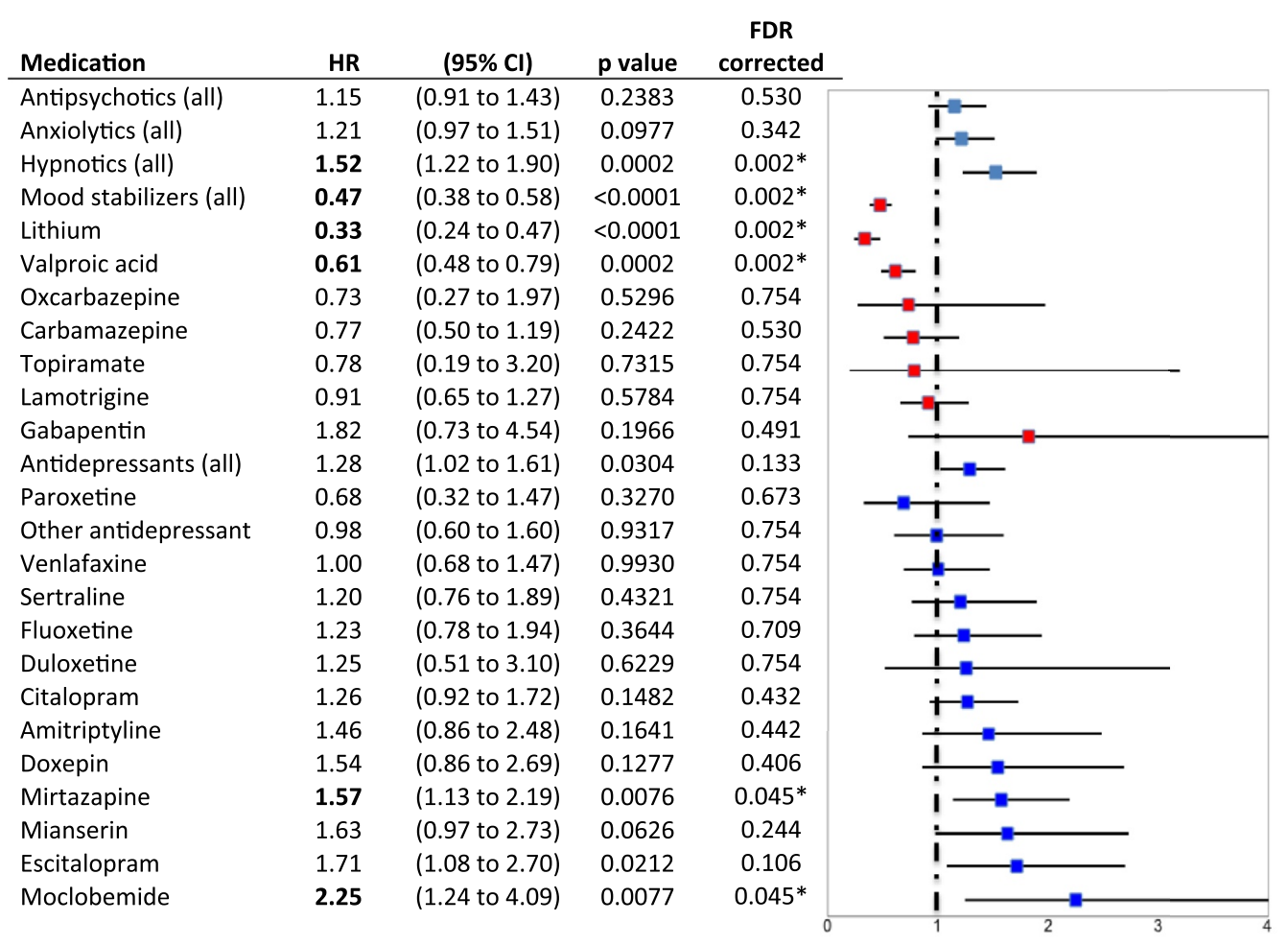

Figure 1. Suicide mortality analysis. Legend: Hazard ratios, 95\% confidence intervals, nominal p-values and Benjamini-Hochberg false discovery rate (FDR) corrected p-values for risk of suicide mortality for use vs. non-use of different medications. P-values still significant after correction for multiple comparisons at $\mathrm{p}<0.05$ level are marked with an *.

\section{Results}

The total cohort population consisted of 18,018 individuals that cumulated 129,740 person-years of follow-up. These individuals had a diagnosis of bipolar disorder, no previous diagnosis of schizophrenia, were alive at cohort entry date (CED) and their CED was before 31.12.2012. The mean follow-up time for the cohort was 7.2 years. During the follow-up, 477 suicide events were recorded. The incident cohort consisted of 2,074 individuals, for whom 32 suicides were registered during the whole follow-up time (14,040 person years). These data are shown in Table 1.

Risk associations for completed suicide with different pharmacotherapies are presented in Fig. 1. Amount of users per medication used during follow-up (not censoring 30 days after the beginning of a hospitalization) is shown in Supplementary Table 1. Of all the psychotropic medications, use of lithium was associated with the lowest and use of valproic acid with second lowest risk of suicide mortality (lithium: HR $0.33,95 \%$ CI 0.24 to $0.47, \mathrm{p}<0.001$ and valproic acid: HR $0.61,95 \% 0.48$ to $0.79, \mathrm{p}<0.001$ ). Results for other mood stabilizers and antipsychotics (data not shown) were not statistically significant. No risk associations for the incident cohort reached statistically significant thresholds, but the rank order was similar to the total cohort. Incident cohort results are reported in Supplementary Table 2.

Although in general, antidepressant treatment had a non-significant (after FDR-correction) increase in risk for suicide mortality in comparison to non-use of antidepressants (HR 1.28, 95\% CI 1.02 to 1.61 , $\mathrm{p}=0.03$, FDR corrected $\mathrm{p}=0.133$ ), the association for risk of suicide increased and turned significant as the number of times an antidepressant regimen had been previously prescribed increased. Compared to persons who had received none or were on their first antidepressant treatment, persons who were on their second antidepressant treatment had more than 2-fold increased risk of completed suicide (HR 2.17, 95\% CI 1.69 to $2.79, \mathrm{p}<0.001$ ) and the ones with at least two prior treatments had more than 3-fold higher risk (HR 3.31, 95\% CI 2.52 to $4.34, \mathrm{p}<0.0001$ ).

The individual antidepressants associated with the highest suicide mortality risk were moclobemide (HR 2.25, 95\% CI $1.24-4.09$, p < 0.008 ) and mirtazapine (HR $1.57,95 \%$ CI 1.13 to 2.19 , p < 0.008). The risk associations for other antidepressants did not reach statistical significance when corrected for multiple comparisons.

Use of hypnotics was associated with a significantly increased risk of suicide (HR 1.52, 95\% CI 1.22 to $1.90, \mathrm{p}=0.0002$ ). Hazard ratios for use of anxiolytics were higher than for non-use, but did not reach statistical significance (HR 1.21, 95\% CI 0.97 to $1.51, \mathrm{p}<0.1$ ). No data on individual hypnotics or anxiolytics was available for analysis.

In order to control for protopathic bias, the groupwise effects of treatments were reanalyzed by omitting all periods within the first 30 days after initiation of a new treatment (Table 2). The results for this analysis were in line with the original analyses, in regard to use of mood stabilizers still being associated with a significantly lower risk (56\%) of suicide mortality (HR $0.44,95$ CI 0.34 to 0.56 , p $<0.0001$ ) and use of hypnotics with a significantly higher $(61 \%)$ risk of suicide mortality (HR 1.61, 95\% CI 1.24 to 2.09 , $\mathrm{p}<0.0004$ ).

Mood stabilizers as a group were associated with the lowest risk for suicide mortality, so use of specific mood stabilizers were compared against the use of valproic acid as a reference (Table 3). The hazard ratio for suicide mortality was $42 \%$ lower for lithium (HR 0.58 , $95 \%$ CI

Table 2

Group level suicide mortality analysis, corrected for protopathic bias.

\begin{tabular}{llll}
\hline Covariates & HR estimate & Confidence interval & p-value \\
\hline anxiolytics & 1.19 & $(0.92-1.55)$ & 0.19 \\
hypnotics & 1.61 & $(1.24-2.09)$ & $0.0003^{*}$ \\
mood stabilizers & 0.44 & $(0.34-0.56)$ & $<0.0001^{*}$ \\
antidepressants & 1.13 & $(0.87-1.46)$ & 0.36 \\
antipsychotics & 1.17 & $(0.90-1.52)$ & 0.24
\end{tabular}

Legend: Analysis to correct for protopathic bias, in which the first 30 days after initiation of any new treatment were omitted from the analysis. The table shows hazard ratios, 95\% confidence intervals and nominal p-values for the risk of suicide mortality when using different medication groups versus not using the same medication group. P-values still significant after FDR correction for multiple comparisons at $\mathrm{p}<0.05$ level are marked with an *. 
Table 3

Head-to-head comparison of mood stabilizers.

\begin{tabular}{llll}
\hline Covariates & HR estimate & Confidence interval & p-value \\
\hline valproic acid & reference & reference & reference \\
lithium & 0.58 & $(0.39-0.86)$ & $0.00651^{*}$ \\
lamotrigine & 1.55 & $(1.05-2.29)$ & 0.02716 \\
carbamazepine & 1.25 & $(0.76-2.07)$ & 0.38366 \\
oxcarbazepine & 1.10 & $(0.35-3.51)$ & 0.87040 \\
gabapentin & 2.85 & $(0.89-9.10)$ & 0.07764 \\
topiramate & 1.03 & $(0.14-7.44)$ & 0.97790 \\
poly use of mood stabilizers & 0.41 & $(0.24-0.69)$ & $0.00095^{*}$ \\
no use of mood stabilizer & 1.65 & $(1.27-2.15)$ & $0.00018^{*}$ \\
\hline
\end{tabular}

Legend: Hazard ratios, 95\% confidence intervals and p-values for risk of suicide mortality for different mood stabilizers as compared to use of valproic acid. No use of mood stabilizer refers to times when a person is not using a mood stabilizer, use of any other medication is allowed. Nominal p-values are displayed. P-values still significant after FDR correction for multiple comparisons at $\mathrm{p}<$ 0.05 level are marked with an *.

0.39 to $0.86, \mathrm{p}<0.007), 59 \%$ lower for poly use of mood stabilizers (HR $0.41,95 \%$ CI 0.24 to $0.69, \mathrm{p}<0.001$ ) and $65 \%$ higher for no use of mood stabilizers (HR 1.65, 95\% CI 1.27 to 2.15 , p $<0.00018$ ) as compared to use of valproic acid. Results for other mood stabilizers did not reach statistical significance.

Some demographic factors were also associated with suicide risk (Table 4). Our study corroborated also for bipolar patients the general finding (Hansson et al., 2018) that male sex is associated with a significantly increased risk of suicide mortality (HR 2.08, 95\% CI 1.73 to $2.52, \mathrm{p}<0.0001)$. We also replicated the previous findings that geriatric patients (age $>70$ ) had a lower risk of suicide (HR $0.22,95 \% \mathrm{CI}$ 0.12 to $0.41, \mathrm{p}<0.001$ ). Time elapsed from initial diagnosis of bipolar disorder seemed to trend with a decreased risk of suicide mortality, although these results did not reach statistical significance (when 0 - 5 years elapsed from initial diagnosis was used as the reference point, 5 10 years elapsed from initial diagnosis was associated with a $24 \%$ reduction in risk and $>10$ years from initial diagnosis with a $35 \%$ reduction in risk of committing suicide).

\section{Discusson}

This observational study suggests that use of lithium or valproic acid

Table 4

Analysis of sex, age at CED, calender year of CED and time since diagnosis in the association between suicide mortality and antidepressant treatment in bipolar disorder.

\begin{tabular}{lll}
\hline Covariates & HR $(95 \% \mathrm{CI})$ & p value \\
\hline Sex & & \\
female & reference & reference \\
male & $1.95(1.62$ to 2.36$)$ & $<0.0001^{*}$ \\
Age at CED & reference & reference \\
$<30$ & $0.92(0.70$ to 1.21$)$ & 0.56492 \\
$30-49$ & $1.00(0.75$ to 1.33$)$ & 0.98603 \\
$50-69$ & $0.30(0.15$ to 0.55$)$ & $0.00012^{*}$ \\
$\geq 70$ & & \\
Calendar year of CED & reference & reference \\
$1996-1999$ & $0.73(0.57$ to 0.94$)$ & 0.01549 \\
$2000-2003$ & $0.48(0.36$ to 0.64$)$ & $<0.0001^{*}$ \\
$2004-2007$ & $0.52(0.38$ to 0.72$)$ & $<0.0001^{*}$ \\
$2008-2012$ & & \\
Time since diagnosis (years) & reference & reference \\
$0-5$ & $0.76(0.53$ to 1.10$)$ & 0.14644 \\
$5-10$ & $0.65(0.37$ to 1.15$)$ & 0.13868 \\
$>10$ & & \\
\hline
\end{tabular}

Legend: Hazard ratios, 95\% confidence intervals and p-values for risk of suicide mortality. CED = Cohort entry date. P-values still significant after FDR correction for multiple comparisons at $\mathrm{p}<0.05$ level are marked with an *. is associated with the lowest risk of suicide mortality in this group of Finnish patients hospitalized at least once for bipolar disorder, indicating that they are the most effective medications in preventing the worst outcome in bipolar disorder, suicide. These results are in accordance with previous studies (Tondo et al., 2001, Tondo and Baldessarini, 2000, Lauterbach et al., 2008). For example, a systematic review and meta-analysis of 48 randomized trials $(n=6,674)$, although three times smaller than the current study in population, revealed that lithium was much more effective than placebo in reducing completed suicides in mood disorders (odds ratio 0.13 , $95 \%$ CI 0.03 to 0.66) (Cipriani et al., 2013).

Secondly, although there is a long standing body of evidence indicating that lithium has specific anti-suicidal properties, there are still insufficient large sample studies analyzing and comparing lithium with newer treatments like anticonvulsants and antipsychotics in preventing mortality due to suicide (Schaffer et al., 2015), although the existing studies so far seem to largely be in favor of lithium (Cipriani et al., 2013, Søndergård et al., 2008). The randomized trial "BALANCE" $(\mathrm{n}=330)$ analyzed valproate and lithium both in monotherapy and in combination for relapse prevention in bipolar I disorder (Geddes et al., 2010). The results of that trial showed that lithium and valproate combination and lithium monotherapy seemed to be better than valproate monotherapy. Another double-blind clinical trial $(\mathrm{n}=98)$ analyzed suicide events and attempts between lithium and valproate users in a sample of high-risk suicide attempters with bipolar disorder, but did not find any differences in effectiveness between the medications (Oquendo et al., 2011). However, yet another study with 12,662 Oregon Medicaid patients diagnosed with bipolar disorder showed a higher ratio of suicide attempts with the use of valproate than with lithium, but not in terms of completed suicides (Collins and McFarland, 2008). In the current study, use of lithium was associated with a markedly lower risk for suicide than use of valproic acid, when compared against each other. Nevertheless, both valproic acid and lithium were associated with a markedly decreased risk for suicide. Although lithium was more effective, valproic acid is considered to have some advantages over lithium, as it is often better tolerated, can be prescribed in a loading-dose strategy and might thus overall have a broader scope in the treatment of bipolar disorder. In general, use of any mood stabilizer (as a groupwise effect) was associated with a reduced risk of committing suicide. This result does likely not stem from mere confounding, as no such positive association was observed for use of antipsychotics, which like mood stabilizers, are also often reserved for the more seriously ill patients.

The usage of antidepressants in bipolar disorder has been substantially under-studied, especially in comparison to their use in unipolar depression (Vieta and Garriga, 2016). Our results show that use of certain antidepressants might in some cases be associated with an increased risk for suicide mortality in patients hospitalized at least once for bipolar disorder, which is in accordance with previous studies (Kessing et al., 2005, McElroy et al., 2006). For instance, in another observational study, a very similar association was found between suicidal behavior and previous antidepressant trials in patients with bipolar disorder (OR 1.29, $\mathrm{p}=0.02$ ) (Undurraga et al., 2012). Also, a study by Kessing et al showed a robust increase in suicide rate (Risk ratio 6.07) in bipolar patients who had purchased antidepressants vs. those who had not (Kessing et al., 2005). By contrast, a large body of research about antidepressants has demonstrated them to be a protective factor in unipolar depression or in the general population for both suicidal behavior and mortality (Reseland et al., 2006), especially after starting to favor the use of selective serotonin re-uptake inhibitors (SSRIs) instead of the more toxic tricyclic antidepressants (TCAs) (Bramness et al., 2007). For example, it was found that suicide rates declined by $13.5 \%$ in the United States from 1985 to 1999, when at the same time antidepressant prescriptions increased over 4-fold, mostly due to SSRIs prescription. The study reported that both prescription rates of SSRIs and other second-generation antidepressants were 
significantly inversely associated with suicide rates (Grunebaum et al., 2004). As antidepressant use in general was not significantly associated with increased risk of suicide (when corrected for multiple comparisons) in our study, it would seem likely that the observed increase in risk as the number of antidepressant treatments increased is skewedness caused by confounding by indication. The individuals with a larger number of antidepressant treatment attempts are likely patients more often relapsing into depression or not benefitting from the first or second antidepressant treatment attempt, and thus in general at a greater risk for suicide.

A previous Swedish study analyzed patients with bipolar disorder who started an antidepressant treatment and had not had antidepressant treatment during the previous year $(n=3,240)$. The main results were that a great percentage of the patients (35\%) were treated with antidepressant monotherapy and that antidepressant monotherapy was associated with an increased risk of treatment-emergent mania (HR 2.83, 95\% CI 1.12 to 7.19 ) (Viktorin et al., 2014). In addition to this, in that same study, it was shown that there was no risk of mania when patients received an antidepressant and a mood stabilizer (lithium, valproate or lamotrigine) at the same time. This prevention of treatment emergent mania could account for part the of association of lowered risk of suicide observed in our study for patients using mood stabilizers such as lithium and valproic acid, although at least lithium has been shown to reduce the risk of suicide both in bipolar and unipolar patients by itself (Cipriani et al., 2005).

The high association between moclobemide and suicides might at least in part be explained by confounding, as this drug is sometimes prescribed to patients after non-adherence to other psychotropics, due it being considered to have lesser side-effects than other MAO-A inhibitors, or being prescribed to patients after several failed attempts with other antidepressants. On the other hand, moclobemide is sometimes also considered to be one the least effective of the antidepressants in treating depression, as was also the case in our previous study estimating the real-world effectiveness of antidepressants in preventing hospitalization in major unipolar depression patients, where moclobemide had the highest hazard ratio for re-hospitalization (Tiihonen et al., 2017). Thus, the increased hazard ratio observed for moclobemide could in part also be due to its ineffectiveness in treating depressive symptoms.

Finally, mood stabilizers, especially lithium and valproic acid seem to be associated with a markedly reduced risk of suicide in bipolar disorder. Despite of this, antidepressants are still widely prescribed as monotherapy to treat bipolar disorder, even for young patients, who are considered to be at a high risk for suicide due to their young age and close temporal proximity to the onset of the disorder (Post et al., 2003, Simon et al., 2004, Shi et al., 2004). This is somewhat understandable given the fact that especially lithium may cause unwanted side-effects for some patients and requires active monitoring and follow-ups. However, our study implies that this antidepressant monotherapy trend might lead to increased suicide rates in bipolar patients and should be more extensively studied, before this treatment regime is further committed to. Further, because suicide risk in bipolar disorder is high at onset of disease and especially for young patients, this group of patients might actually benefit most from the lower risk of suicide mortality associated with use of lithium or valproic acid (Vieta et al., 2018). In our study, use of hypnotics was also associated with an increased risk of suicide. Although the result may be due to residual confounding, it is in line with a previous study by Kessing et al. (Kessing et al., 2005). As these drugs along with anxiolytics might inhibit impulse control, possibly even after their hypnotic effect wears off, they might lead to increased translation from suicidal ideations to suicidal behaviors (Dodds, 2017, Sun et al., 2016). Patients at high risk for suicide using these medications should therefore be closely monitored.

\subsection{Limitations}

Our study only involved individuals who have been hospitalized due to bipolar disorder. As such, patients with a milder course of illness, such as some type II bipolar patients might not be well represented in this sample. However, the Finnish national registries are very comprehensive and it is likely that we have caught nigh all of the patients hospitalized due to bipolar disorder in Finland. The universal healthcare and high quality of psychiatric care provided also increases the likelihood that schizophrenia spectrum disorders were comprehensively excluded from the sample. We did not analyze the effect of other psychiatric comorbidities, such as substance abuse disorders, anxiety disorders or personality disorders in this study, which is a marked limitation. It must be noted that it is possible that some or all of the association with increased risk observed in the current study could be due to confounding by indication or protopathic bias, as our analysis for protopathic bias would suggest. Hence, further studies are warranted, and rather than pointing at an increased risk of suicide due to the effect of antidepressant medications, our results could be interpreted in the sense that the patients who received antidepressants were those who were at highest risk of suicide (medication initiated due to greater depressive symptom severity). As suicide is a one-time event, withinindividual analyses capable of correcting for this sort of confounding by indication could not be performed. However, as this result remains somewhat unclear, this study does warrant for closer clinical monitoring of bipolar patients using antidepressants and at an otherwise high risk for suicide.

\section{Conclusions}

According to our data, use of mood stabilizers, primarily lithium, but also valproic acid were associated with a markedly lower risk of dying by suicide among patients with bipolar disorder. Use of hypnotics in general was associated with an increased risk of suicide.

To conclude, clinicians should continue prescribing lithium or valproic acid to suitable bipolar patients at high risk for suicide with risk factors including but not limited to close proximity to onset of disorder, previous suicide attempts, younger age, comorbid anxiety disorders, relatives with bipolar disorder and previous manic or hypomanic episodes. Lithium should be the primary choice, but valproic acid might be suitable for situations in which quick loading-doses are required or sideeffects are a major concern, such as for the very young and the elderly. Use of hypnotics in patients at an increased risk for suicide should trigger the need for closer monitoring for suicidal signals.

\section{Disclosures/Conflicts of interest}

M. Lähteenvuo is a shareholder and board member at Genomi Solutions ltd. and DNE ltd.. He has also received research grants, honoraria or awards from Boehringer-Ingelheim ltd., Sunovion ltd., Janssen-Cilag and Orion Pharma ltd. and has been working as a coordinator for a research project funded by the Stanley Foundation. D. Antolín Concha reports no disclosures. P. Vattulainen is employed by EPID Research, which is a contract research organization that performs commissioned pharmacoepidemiological studies and thus its employees have been and currently are working in collaboration with several pharmaceutical companies. A. Tanskanen, H. Taipale and J. Tiihonen have participated in research projects funded by Eli Lilly and JanssenCilag with grants paid to the Karolinska Institutet.E. Vieta has received grants or served as consultant, advisor, or continuing medical education speaker for AB-Biotics, Aequus, Adamed, Alexza, Allergan, Almirall, AstraZeneca, Bial, Bristol-Myers Squibb, Dainippon Sumitomo Pharma, Elan, Eli Lilly, Esteve, Ferrer, Forest Research Institute, Gedeon Richter, GlaxoSmithKline, Janssen-Cilag, Jazz, Johnson \& Johnson, Lundbeck, Merck, Novartis, Organon, Otsuka, Pfizer, Pierre-Fabre, Rovi, Qualigen, Roche, Sanofi, Servier, Schering-Plough, Shire, Solvay, Sunovion, 
Takeda, Telefónica, Teva, the Spanish Ministry of Science and Innovation, the Seventh European Framework Programme, the Stanley Medical Research Institute, United Biosource Corporation, and Wyeth. J. Tiihonen has served as a consultant to the Finnish Medicines Agency (Fimea) and European Medicines Agency (EMA); has received lecture fees from Eli Lilly, Janssen-Cilag, Lundbeck, and Otsuka; and grants from Stanley Foundation and Sigrid Jusélius Foundation.

\section{Author contribution statement}

Concept and design: ML, DAC, JT, HT, AT.

Acquisition, analysis, or interpretation of data: All authors.

Drafting of the manuscript: DAC, ML

Critical revision of the manuscript for important intellectual content: All authors.

Statistical analysis: HT, AT, PV

Obtained funding: ML, JT.

\section{Funding}

This work was supported by by the Finnish Institute for Health and Welfare by a grant given to Niuvanniemi hospital. Additional support was obtained from the Finnish Medical Association and Emil Aaltonen foundation (personal working grants to Lähteenvuo).

\section{Role of the funding source}

The funding source had on role in the planning or the implementation of this study.

\section{Acknowledgements}

The authors would like to thank ms. Aija Räsänen for excellent secreterial assistance.

\section{Supplementary materials}

Supplementary material associated with this article can be found, in the online version, at doi:10.1016/j.jad.2020.09.016.

\section{References}

Clemente, AS, Diniz, BS, Nicolato, R, et al., 2015. Bipolar disorder prevalence: a systematic review and meta-analysis of the literature. Rev. Bras. Psiquiatr. 37, 155-161.

Angst, J, 1998. The emerging epidemiology of hypomania and bipolar II disorder. J. Affect. Disord. 50, 143-151.

Akiskal, HS, Bourgeois, ML, Angst, J, et al., 2000. Re-evaluating the prevalence of and diagnostic composition within the broad clinical spectrum of bipolar disorders. J. Affect. Disord. 59, S5-S30.

Crump, C, Sundquist, K, Winkleby, MA, Sundquist, J, 2013. Comorbidities and mortality in bipolar disorder: a Swedish national cohort study. JAMA Psychiatry 70 (9), 931-939.

Medici, CR, Videbech, P, Gustafsson, LN, et al., 2015. Mortality and secular trend in the incidence of bipolar disorder. J. Affect. Disord. 183, 39-44.

Hayes, JF, Miles, J, Walters, K, et al., 2015. A systematic review and meta-analysis of premature mortality in bipolar affective disorder. Acta Psychiatr Scand. 131, 417-425.

Collins, JC, McFarland, BH, 2008. Divalproex, lithium, and suicide among Medicaid patients with bipolar disorder. J. Affect. Disord. 107 (1-3), 23-28.

Vieta, E, Langosch, JM, Figueira, ML, et al., 2013 Sep. Clinical management and burden of bipolar disorder: results from a multinational longitudinal study (WAVE-bd). Int. J. Neuropsychopharmacol. 16 (8), 1719-1732.

Kessing, LV, Vradi, E, Andersen, PK, 2016. Nationwide and population-based prescription patterns in bipolar disorder. Bipolar Disord. 18 (2), 174-182.

Karanti, A, Kardell, M, Lundberg, U, Landén, M, 2016. Changes in mood stabilizer prescription patterns in bipolar disorder. J. Affect Disord. 195, 50-56.

Rhee, TG, Olfson, M, Nierenberg, AA, Wilkinson, ST, 2020. 20-Year trends in the pharmacologic treatment of bipolar disorder by psychiatrists in outpatient care settings. Am. J. Psychiatry 177 (8), 706-715.

Vieta, E, Berk, M, Schulze, TG, et al., 2018. Bipolar disorders. Nat. Rev. Dis. Primers 4, 18008.

Carlborg, A, Ferntoft, L, Thuresson, M, et al., 2015. Population study of disease burden, management, and treatment of bipolar disorder in Sweden: a retrospective observational registry study. Bipolar Disord. 17, 76-85.

Ösby, U, Brandt, L, Correia, N, et al., 2001. Excess mortality in bipolar and unipolar disorder in Sweden. Arch. Gen. Psychiatr. 58, 844-850.

Kessing, LV, Søndergård, L, Kvist, K, Andersen, PK, 2005. Suicide risk in patients treated with lithium. Arch. Gen. Psychiatry 62 (8), 860-866.

Smith, KA, Cipriani, A, 2017. Lithium and suicide in mood disorders: updated meta-review of the scientific literature. Bipolar Disord. 19 (7), 575-586.

Cipriani, A, Pretty, H, Hawton, K, et al., 2005. Lithium in the prevention of suicidal behavior and all-cause mortality in patients with mood disorders: a systematic review of randomized trials. Am. J. Psychiatry 162, 1805-1819.

Cipriani, A, Hawton, K, Stockton, S, et al., 2013. Lithium in the prevention of suicide in mood disorders: updated systematic review and meta-analysis. BMJ 346, f3646.

Tondo, L, Baldessarini, RJ, 2000. Reduced suicide risk during lithium maintenance treatment. J. Clin. Psychiatry 61 (suppl 9), 97-104.

Tondo, L, Hennen, J, Baldessarini, RJ, 2001. Lower suicide risk with long-term lithium treatment in major affective illness: a meta-analysis. Acta Psychiatr. Scand. 104, 163-172.

Öhlund, L, Ott, M, Oja, S, et al., 2018. Reasons for lithium discontinuation in men and women with bipolar disorder: a retrospective cohort study [published correction appears in BMC Psychiatry. 2018 Oct 3;18(1):322]. BMC Psychiatry 18 (1), 37 Published 2018 Feb 7.

Søndergård, L, Lopez, AG, Andersen, PK, Kessing, LV, 2008. Mood-stabilizing pharmacological treatment in bipolar disorders and risk of suicide. Bipolar Disord. 10 (1) 87-94.

Smith, EG, Søndergård, L, Lopez, AG, Andersen, PK, Kessing, LV, 2009. Association between consistent purchase of anticonvulsants or lithium and suicide risk: a longitudinal cohort study from Denmark, 1995-2001. J. Affect. Disord. 117 (3), 162-167.

Song, J, Sjölander, A, Joas, E, et al., 2017. Suicidal behavior during lithium and valproate treatment: a within-individual 8-year prospective study of 50,000 patients with bipolar disorder. Am. J. Psychiatry 174 (8), 795-802.

Ferrer, P, Ballarín, E, Sabaté, M, et al., 2014. Antiepileptic drugs and suicide: a systematic review of adverse effects. Neuroepidemiology 42 (2), 107-120.

Collins, JC, McFarland, BH, 2008. Divalproex, lithium, and suicide among Medicaid patients with bipolar disorder. J. Affect. Disord. 107 (1-3), 23-28.

Geddes, JR, Goodwin, GR, Rendell, J, et al., 2010. Lithium plus valproate combination therapy versus monotherapy for relapse prevention in bipolar I disorder (BALANCE): a randomised open-label trial. Lancet 375 (9712), 385-395.

Oquendo, MA, Galfalvy, HC, Currier, D, et al., 2011. Treatment of suicide attempters with bipolar disorder: a randomized clinical trial comparing lithium and valproate in the prevention of suicidal behavior. Am. J. Psychiatry 168 (10), 1050-1056.

Baldessarini, RJ, Tondo, L, Vázquez, GH, 2019. Pharmacological treatment of adult bipolar disorder. Mol. Psychiatry 24 (2), 198-217.

Tondo, L, Baldessarini, RJ, 2018. Antisuicidal effects in mood disorders: are they unique to lithium? Pharmacopsychiatry 51 (5), 177-188. https://doi.org/10.1055/a-05967853.

Marangell, LB, Dennehy, EB, Wisniewski, SR, et al., 2008. Case-control analyses of the impact of pharmacotherapy on prospectively observed suicide attempts and completed suicides in bipolar disorder: findings from STEP-BD. J. Clin. Psychiatry 69 (6), 916-922. https://doi.org/10.4088/jcp.v69n0605.

Schaffer, A, Isometsä, ET, Tondo, L, et al., 2015. Epidemiology, neurobiology and pharmacological interventions related to suicide deaths and suicide attempts in bipolar disorder: Part I of a report of the international society for bipolar disorders task force on suicide in bipolar disorder. Aust. N. Z. J. Psychiatry 49 (9), 785-802. https://doi. org $/ 10.1177 / 0004867415594427$.

Viktorin, A, Lichtenstein, P, Thase, ME, et al., 2014. The risk of switch to mania in patients with bipolar disorder during treatment with an antidepressant alone and in combination with a mood stabilizer. Am. J. Psychiatry 171, 1067-1073.

Pacchiarotti, I, Bond, DJ, Baldessarini, RJ, et al., 2013. The International Society fo Bipolar Disorders (ISBD) task force report on antidepressant use in bipolar disorders. Am. J. Psychiatry 170 (11), 1249-1262.

Vieta, E, 2014 Oct. Antidepressants in bipolar I disorder: never as monotherapy. Am. J. Psychiatry 171 (10), 1023-1026.

Tanskanen, A, Taipale, H, Koponen, M, et al., 2017. Drug exposure in register-based research - An expert-opinion based evaluation of methods. PLoS One 12 (9), e0184070.

Tanskanen, A, Taipale, H, Koponen, M, et al., 2015. From prescription drug purchases to drug use periods - a second generation method (PRE2DUP). BMC Med. Inform Decis. Mak. 15, 21

Taipale, H, Tanskanen, A, Koponen, M, et al., 2016. Agreement between PRE2DUP register data modeling method and comprehensive drug use interview among older persons. Clin. Epidemiol. 8, 363-371.

Tiihonen, J, Tanskanen, A, Hoti, F, et al., 2017. Pharmacological treatments and risk of readmission to hospital for unipolar depression in Finland: a nationwide cohort study. Lancet Psychiatry 4 (7), 547-553.

Hansson, C, Joas, E, Pålsson, E, et al., 2018. Risk factors for suicide in bipolar disorder: a cohort study of 12850 patients. Acta Psychiatr. Scand. 138 (5), 456-463.

Tondo, L, Baldessarini, RJ, 2000. Reduced suicide risk during lithium maintenance treatment. J. Clin. Psychiatry 61 (suppl 9), 97-104.

Lauterbach, E, Felber, W, Müller-Oerlinghausen, B, et al., 2008. Adjunctive lithium treatment in the prevention of suicidal behaviour in depressive disorders: a randomised, placebo-controlled, 1-year trial. Acta Psychiatr. Scand. 118 (6), 469-476.

Vieta, E, Garriga, M, 2016. Adjunctive antidepressants in bipolar depression. Lancet Psychiatry 3 (12), 1095-1096.

McElroy, SL, Kotwal, R, Kaneria, R, et al., 2006. Antidepressants and suicidal behavior in bipolar disorder. Bipolar Disord. 8, 596-617.

Undurraga, J, Baldessarini, RJ, Valenti, M, et al., 2012. Suicidal risk factors in bipolar I and II disorder patients. J. Clin. Psychiatr. 73, 778-782. 
Reseland, S, Bray, I, Gunnell, D, 2006. Relationship between antidepressant sales and secular trends in suicide rates in the Nordic countries. Br. J. Psychiatry 188, 354-358.

Bramness, JG, Walby, FA, Tverdal, A, 2007. The sales of antidepressants and suicide rates in Norway and its counties 1980-2004. J. Affect. Disord. 102, 1-9.

Grunebaum, MF, Ellis, SP, Li, S, et al., 2004. Antidepressants and suicide risk in the United States, 1985-1999. J. Clin. Psychiatry 65, 1456-1462.

Tiihonen, J, Tanskanen, A, Hoti, F, et al., 2017. Pharmacological treatments and risk of readmission to hospital for unipolar depression in Finland: a nationwide cohort study. Lancet Psychiatry 4 (7), 547-553.

Post, RM, Leverich, GS, Altshuler, LL, et al., 2003. An overview of recent findings of the Stanley Foundation Bipolar Network (Part 1). Bipolar Disord. 5, 310-319.

Simon, N, Otto, M, Weiss, R, et al., 2004. Pharmacotherapy for bipolar disorder and comorbid conditions: baseline data from STEP-BD. J. Clin. Psychopharmacol. 24, 512-520.

Shi, L, Thiebaud, P, McCombs, JS, 2004. The impact of unrecognized bipolar disorder for patients treated with depression with antidepressants in the fee-for-services California Medicaid (Medi-Cal) programme. J. Affect. Disord. 82, 373-383.

Vieta, E, Salagre, E, Grande, I, et al., 2018. Early intervention in bipolar disorder. Am. J. Psychiatry 175 (5), 411-426.

Dodds, TJ, 2017. Prescribed benzodiazepines and suicide risk: a review of the literature Prim Care Companion CNS Disord. 19 (2), 16 r02037.

Sun, Y, Lin, CC, Lu, CJ, et al., 2016. Association between zolpidem and suicide:a nationwide population-based case-control study. Mayo Clin. Proc. 91 (3), 308-315. 